OPEN ACCESS

Edited by:

Bernard Jimmy Hanseeuw, Cliniques Universitaires Saint-Luc,

Belgium

Reviewed by:

Tracy Fischer,

Tulane University, United States

Elizabeth Kuhn,

Institut National de la Santé et de la

Recherche Médicale (INSERM),

France

${ }^{*}$ Correspondence:

Ana Luisa Sosa-Ortiz drasosa@hotmail.com

Received: 10 July 2021 Accepted: 05 October 2021 Published: 28 October 2021

Citation:

Flores-Vázquez JF

Ramírez-García G,

Marrufo-Meléndez OR, Alcalá-Lozano R, Lietz MP,

Rodríguez-Agudelo $Y$ Acosta-Castillo Gl, Renken RJ, Aleman A, Enriquez-Geppert S and Sosa-Ortiz AL (2021) Anosognosia

in Amnestic Mild Cognitive

Impairment Is Related to Diminished Hippocampal Volume Comparable to Alzheimer's Disease Dementia:

Preliminary MRI Findings.

Front. Aging Neurosci. 13:739422.

doi: 10.3389/fnagi.2021.739422

\section{Anosognosia in Amnestic Mild Cognitive Impairment Is Related to Diminished Hippocampal Volume Comparable to Alzheimer's Disease Dementia: Preliminary MRI Findings}

\author{
Juan Francisco Flores-Vázquez 1,2,3,4, Gabriel Ramírez-García5, \\ Oscar René Marrufo-Meléndez 6 , Ruth Alcalá-Lozano7, Morten Peter Lietz ${ }^{3,4}$, \\ Yaneth Rodríguez-Agudelo8, Gilberto Isaac Acosta-Castillo',2, Remco J. Renken ${ }^{4}$, \\ Andre Aleman ${ }^{3,4}$, Stefanie Enriquez-Geppert ${ }^{3,4}$ and Ana Luisa Sosa-Ortiz',2*
}

\begin{abstract}
' Programa de Maestría y Doctorado en Ciencias Médicas, Odontológicas y de la Salud, Universidad Nacional Autónoma de México, Mexico City, Mexico, ${ }^{2}$ Laboratorio de Demencias, Instituto Nacional de Neurología y Neurocirugía Manuel Velasco Suárez, Mexico City, Mexico, ${ }^{3}$ Department of Clinical and Developmental Neuropsychology, University of Groningen, Groningen, Netherlands, ${ }^{4}$ Section of Cognitive Neuropsychiatry, Department of Biomedical Sciences of Cells and Systems, University of Groningen, Groningen, Netherlands, ${ }^{5}$ Laboratorio de Neuropsicología, Departamento de Fisiología, Facultad de Medicina, Universidad Nacional Autónoma de México, Mexico City, Mexico, ${ }^{6}$ Unidad de Neuroimagen, Instituto Nacional de Neurología y Neurocirugía Manuel Velasco Suárez, Mexico City, Mexico, ${ }^{7}$ Subdirección de Investigaciones Clínicas, Instituto Nacional de Psiquiatría Ramón de la Fuente Muñiz, Mexico City, Mexico, ${ }^{8}$ Departamento de Neuropsicología, Instituto Nacional de Neurología y Neurocirugía Manuel Velasco Suárez, Mexico City, Mexico
\end{abstract}

Although the presence of anosognosia in amnestic mild cognitive impairment (aMCl) may be predictive of conversion to Alzheimer's disease (AD), little is known about its neural correlates in $\mathrm{AD}$ and $\mathrm{aMCl}$. Four different groups were compared using volumetric and diffusion magnetic resonance imaging metrics in regions of interest (hippocampus and cingulum cortex gray matter, cingulum bundle white matter): $\mathrm{aMCl}$ subjects with anosognosia $(n=6)$, aMCl subjects without anosognosia $(n=12), \mathrm{AD}$ subjects with anosognosia $(n=6)$, and AD subjects without anosognosia $(n=9)$. $\mathrm{aMCl}$ subjects with anosognosia displayed a significantly lower gray matter density (GMD) in the bilateral hippocampus than aMCl subjects without anosognosia, which was accounted for by bilateral hippocampal differences. Furthermore, we identified that the mean hippocampal gray matter density of aMCl subjects with anosognosia was not statistically different than that of $A D$ subjects. The groups of $\mathrm{aMCl}$ and $A D$ subjects with anosognosia also displayed a lower GMD in the bilateral cingulum cortex compared to subjects without anosognosia, but these differences were not statistically significant. No statistically significant differences were found in the fractional anisotropy or mean diffusivity of the hippocampus or cingulum between subjects with and without anosognosia in $\mathrm{aMCl}$ or $\mathrm{AD}$ groups. While these findings are derived from a small population of subjects and are in need of replication, they suggest that anosognosia in $\mathrm{aMCl}$ might be a useful clinical marker to suspect brain changes associated with AD neuropathology.

Keywords: mild cognitive impairment, Alzheimer's disease, anosognosia, hippocampus, magnetic resonance imaging 


\section{INTRODUCTION}

Anosognosia is defined as the loss or decline in a subject's awareness of problems in daily functioning, behavior, cognition, or mood (Starkstein, 2014). This condition is frequent throughout the trajectory of Alzheimer's disease (AD), with a prevalence ranging between 20 and $80 \%$, and its presence is linked to increased dependence, reduced adherence to treatment and risk behaviors in patients, increased caregiver distress, and a greater economic burden for families and societies (Turró-Garriga et al., 2013; Starkstein, 2014).

Anosognosia can appear in amnestic mild cognitive impairment (aMCI), a diagnosis that implies a heightened risk for developing $\mathrm{AD}$ dementia, in which memory performance is diminished but autonomy in daily life is preserved (Mak et al., 2015). Interestingly, the presence of anosognosia in aMCI has been associated with underlying brain changes characteristic of $\mathrm{AD}$, and may have a predictive value for further worsening of cognition and progression to $\mathrm{AD}$ dementia (Scherling et al., 2016; Gerretsen et al., 2017; Therriault et al., 2018; Hanseeuw et al., 2020). As only a fraction of aMCI-affected subjects will progress to dementia, the clinical characterization of subjects at a higher risk of developing $\mathrm{AD}$ is a major concern in current research. This, in turn, can support timely interventions before significant neural and functional impairment has taken place. The understanding of the neural changes associated with anosognosia is therefore necessary for the adequate characterization of aMCI and the early stages of AD (Mondragón et al., 2021).

According to a recent systematic review, anosognosia in $\mathrm{AD}$ is associated with a reduction in gray matter density, cerebral blood flow, and metabolism in several regions: the anterior and posterior cingulate cortex, the medial temporal lobe, the inferior, superior and medial frontal gyri, the orbitofrontal cortex, and the insula (Hallam et al., 2020). Most of these regions form part of the default mode network, a large-scale brain network that is altered from the early stages of the AD continuum (Grieder et al., 2018) and is associated with self-related cognition (e.g., introspection and autobiographic memory) (Zamboni et al., 2013). Said systematic review identified that measurement heterogeneity is one of the main limitations in integrating previous clinical studies on anosognosia in $\mathrm{AMCI}$ and $\mathrm{AD}$ dementia.

In clinical research, three methods have been mainly used to measure anosognosia in AD (Hallam et al., 2020). (1) Clinical rating, in which the clinician's judgment is used to rate the level of anosognosia on a scale after an interview with the patient and the caregiver. (2) Patient-informant discrepancy, in which after parallel interviewing of the patient and caregiver, a "discrepancy score" of the patient's symptoms is calculated, and finally. (3) Performance discrepancy, in which the performance of a patient on a neuropsychological test is compared to their own estimation of how well they think they performed on said test. Taking this into consideration, a multi-method magnetic resonance imaging (MRI) morphometric study showed that all three anosognosia measurement methods were independently associated with gray matter atrophy in the medial temporal lobe including the right hippocampus in $\mathrm{AD}$ participants (Tondelli et al., 2018). The consistent involvement of the medial temporal lobe and the hippocampus supports the view that anosognosia is principally caused by a decline in memory processes (such as the autobiographical episodic memory loss typically characterizing $\mathrm{AD})$ that prevents the update of self-knowledge (Morris and Mograbi, 2013; Chavoix and Insausti, 2017). However, the neural substrate of anosognosia in AD is far from being fully elucidated, and there is a need for replication of previous findings along with the development of objective anosognosia measurements (Hallam et al., 2020).

A sound approach for the assessment of anosognosia has been developed under the more general construct of the behavioral dysexecutive syndrome (Godefroy et al., 2010). This syndrome groups twelve symptoms related to disturbances in the executive function brain network (e.g., anosognosia, apathy, irritability, and confabulations) that are frequently observed in several neurocognitive disorders. In mild AD dementia, $86 \%$ of patients have been found to exhibit a behavioral dysexecutive syndrome, with a large effect size when comparing the severity and of anosognosia between AD participants and healthy controls (Godefroy et al., 2014). Along with the definition of the syndrome, a straightforward structured questionnaire has been proposed, the Behavioral Dysexecutive Syndrome Inventory (BDSI), which is applied to an informant who knows the patient well, and aims to measure the frequency and severity of each symptom - including anosognosia (Godefroy et al., 2010).

In light of this evidence, we aimed to provide further evidence on the neural underpinnings of anosognosia in aMCI and AD using the BDSI in a clinical sample. To our knowledge, no previous studies have used this anosognosia measurement to study structural brain changes. We hypothesized that subjects with aMCI or AD dementia with anosognosia would exhibit volumetric and white-matter integrity changes in the bilateral hippocampus and cingulate cortex, relative to aMCI or $\mathrm{AD}$ subjects without anosognosia.

\section{MATERIALS AND METHODS}

\section{Sample and Participants}

Eighteen participants with aMCI and sixteen participants with $\mathrm{AD}$ were recruited from the outpatient consultation of the Dementia Laboratory of the National Institute of Neurology and Neurosurgery of Mexico in Mexico City. aMCI and AD dementia were diagnosed by certified specialists following current clinical criteria (Petersen, 2004; McKhann et al., 2011). Inclusion criteria further consisted of: being between 60 and 76 years of age, a Mini-Mental Score Examination (MMSE) score of 25 or higher in the aMCI group and 16 or higher in the $\mathrm{AD}$ dementia group, and having a knowledgeable informant living with the subject who could answer to the clinical questionnaires. Exclusion criteria consisted of a clinical history suggestive of non-AD dementia, current symptoms suggestive of delirium, major depression, substance-use disorders, or other major neuropsychiatric disorders (apart from aMCI or AD dementia), not being able to complete clinical or neuroimaging assessments, and MRI contraindications. 


\section{Clinical Measurements}

General cognitive functioning was assessed using an adapted version of the MMSE widely used in Mexico (Ostrosky-Solís et al., 2000). For the assessment of anosognosia, a cross-culturally adapted Mexican version (Flores-Vazquez and Sosa-Ortiz, 2016) of the BDSI (Godefroy et al., 2010) was used. Subjects were divided into "anosognosia" or "no-anosognosia" groups if the answer given to the screening questions presented in Table $\mathbf{1}$ was "yes" or "no." Additional analysis taking into consideration the severity and frequency of anosognosia can be consulted in the Supplementary Material.

\section{Magnetic Resonance Imaging Acquisition and Processing}

Magnetic resonance imagings were acquired using a 3 Tesla SIEMENS Skyra scanner (Erlangen, Germany) with a 20ch head coil.

T1-weighted images were obtained using a 3D MPRAGE sequence (TR/TE: 2,300/2.45 ms; FOV: $256 \mathrm{~mm}^{2}$; matrix: $256 \times 256$; voxel size: $1 \mathrm{~mm}^{3}$ ). Preprocessing included denoising and intensity inhomogeneity correction (Manjón et al., 2010; Avants et al., 2011). T1-weighted images were processed using the VBM-FSL toolbox (Douaud et al., 2007; Jenkinson et al., 2012). T1 image processing included brain extraction, tissuetype segmentation, the creation of a study-specific gray matter template, registration of all gray matter images into the template,

TABLE 1 | Anosognosia assessment in the behavioral dysexecutive syndrome inventory (Godefroy et al., 2010).

Screening questions: Does the subject minimize or fail to recognize the limitations or difficulties that they have and the consequences of those limitations in daily life? Does the subject make unrealistic plans? Does the subject think that they can do the same things they used to do even when this is no longer realistic?

Specific questions (used for clarification):

- Does the subject tend to minimize their own cognitive decline, for instance, their memory problems?

- Does the subject tend to minimize their behavioral problems?

- Does the subject tend to minimize their impairments when moving, seeing, or hearing?

- Is the subject indifferent to their impairments although these impairments impact their daily life?

- Does the subject blame their impairments on other people or the situation?

- Does the subject deny their impairments although other people can notice them?

- Does the subject act as if they had no illness and need no help from others?

- Does the subject make unrealistic plans and wrongly thinks they could retake previous activities?

Frequency scoring: 1 = Rarely: less than once a week.

2 = Sometimes = approximately once a week. 3 = Frequently: several times a week, but not every day. 4 = Very frequently = every day $/$ most of the time.

Severity scoring: 1 = Mild: noticeable, few consequences in everyday life. 2 = Moderate: significant and disturbing, but manageable. 3 = Severe: very marked and disturbing, very difficult to manage

${ }^{a}$ If the answer to any of the screening questions is "yes," the anosognosia domain should be examined at depth using the specific questions, and the frequency and severity of the anosognosia domain should be scored
Jacobian modulation, and smoothing. Of note, including the Jacobian modulation step in the processing pipeline handles the variability in the head size of the subjects at a local level, eliminating the need for controlling or correcting for by ICV (Douaud et al., 2007). The gray-matter regions of interest (ROIs) of the bilateral hippocampus and cingulate cortex were defined using the Harvard-Oxford Cortical and Subcortical atlases (Desikan et al., 2006), respectively (Figure 1). First, each ROI was eroded to reduce its size according to the anatomical region into the MNI standard space; then, all ROIs were binarized. These ROIs were used to extract the mean gray matter density (GMD).

Diffusion-weighted images (DWI) were obtained using an echo-planar spin-echo sequence with 64 directions (TR/TE: 5,000/102 ms; FOV: $220 \mathrm{~mm}^{2}$; matrix: $100 \times 100$; voxel size: $2.2 \mathrm{~mm}^{3}$ ). DWI image preprocessing included a first denoising step using a blockwise non-local means filter (Coupé et al., 2008), after which, eddy current and subject movement correction, binary mask creation, and diffusion tensor fitting to obtain the scalar anisotropy and diffusivity maps were completed using the TBSS-FSL toolbox (Smith et al., 2006; Jenkinson et al., 2012). Fractional anisotropy (FA) and mean diffusivity (MD) maps were non-linearly registered to the MNI standard space following the TBSS steps (Smith et al., 2006; Jenkinson et al., 2012). The white-matter ROIs of the bilateral cingulum bundle (Figure 1) were defined using the JHU-DTI-81 White-Matter Labels atlas (Wakana et al., 2007). First, each ROI was eroded to reduce its size according to the anatomical region into the MNI standard space; then, all ROIs were binarized. These ROIs were used to extract the mean FA or MD of the specific white matter tracts.

\section{Statistical Analysis}

Results are presented in means $(M)$, medians (Mdn), interquartile ranges (IQR), and value ranges (Rng). For the description and comparison of demographic and clinical characteristics, MannWhitney $U$ tests were used for analyzing continuous variables (years age, intracranial volume - ICV, and MMSE score), and Chi-square tests for dichotomous variables (sex).

For the group comparisons (aMCI-anosognosia vs. aMCIno-anosognosia; $\mathrm{AD}$-anosognosia vs. $\mathrm{AD}$-no-anosognosia) of the averaged GMD or white matter FA in each ROI, Mann-Whitney $U$ tests were used. An alpha of 0.05 was defined for statistically significant findings. In the case of statistically significant findings, we conducted a False Discovery Rate analysis, and present the Benjamini-Hochberg adjusted $p$ value. The rank-biserial correlation $\left(r_{r b}\right)$ is reported as a non-parametric effect size for the Mann-Whitney $U$ test (Kerby, 2014). Statistical analyses were carried out in the JASP software version 0.14.1 (JASP Team, 2020) and IBM SPSS Statistics version 27 (IBM Corp, 2020).

Additional analysis assessing the correlation of the severity and frequency of anosognosia with the MRI metrics can be consulted in the Supplementary Material.

\section{Ethical Considerations}

This study was approved by the research and ethics committees of the National Institute of Neurology and Neurosurgery of Mexico after independent, blinded review (protocol number: 116/16) 


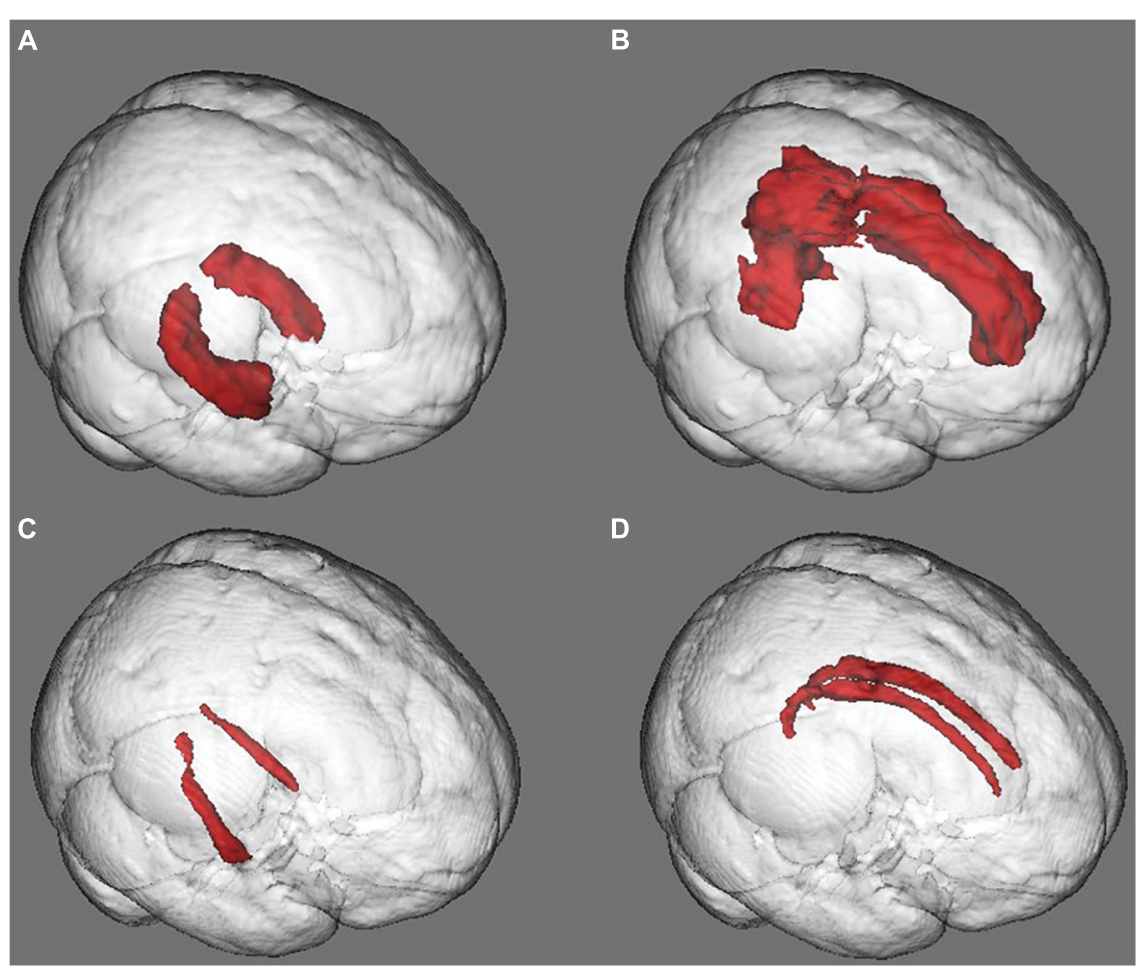

FIGURE 1 | Gray and white matter regions of interest (ROIs). (A) Hippocampal gray matter ROI. (B) Cingulum gray matter ROI. (C) Hippocampal white matter ROI. (D) Cingulum white matter ROI. The morphological or diffusion values were averaged in each ROl for between-group comparisons.

and carried out according to the Declaration of Helsinki. All participating subjects, as well as first-grade family members in the case of subjects with AD dementia, were informed about the study in detail and consented to it.

\section{RESULTS}

\section{Demographic and Clinical Characteristics}

In the aMCI group $(n=18)$, subjects displaying anosognosia ( $n=6$ and 2 female) had a Mdn of 72.0 years of age $(I Q R=3.0)$ and a Mdn of 12.0 years of education $(I Q R=5.3)$, obtained a Mdn MMSE score of $26.5(I Q R=1.8)$, and had a Mdn ICV of 1358.4 $(I Q R=147.0) \mathrm{cm}^{3}$. aMCI subjects not displaying anosognosia $(n=12$ and 9 female) had a Mdn of 69.5 years of age $(I Q R=7.0)$ and a Mdn of 12.0 years of education $(I Q R=7.0)$, obtained a Mdn score of 28.0 in the MMSE $(I Q R=1.3)$, and had a Mdn ICV of $1292.0 \mathrm{~cm}^{3}(I Q R=64.5)$. Between-group differences were not statistically significant in any of these variables, median, ranges, test statistics, and $p$ values are presented in Table 2.

In the $\mathrm{AD}$ group $(n=16)$, subjects displaying anosognosia ( $n=7$ and 5 female) had a Mdn of 72.0 years of age $(I Q R=6.0)$, and a Mdn of 9.0 years of education $(I Q R=3.5)$, obtained a Mdn score of 21.0 in the MMSE $(I Q R=4.0)$, and had a Mdn ICV of $1358.1 \mathrm{~cm}^{3}(I Q R=137.6)$. AD subjects not displaying anosognosia ( $n=9$ and 5 female) had a Mdn of 64.5 years of age $(I Q R=6.0)$ and a Mdn of 12 years of education $(I Q R=7.0)$, obtained a Mdn score of 22.0 in the MMSE $(I Q R=4.0)$, and had a Mdn ICV of $1293.4 \mathrm{~cm}^{3}(I Q R=117.3)$. Between-group differences were not statistically significant, and mean, ranges, test statistics with $p$ values are presented in Table 2 .

\section{Gray Matter Volumetric Comparisons}

In the aMCI group, subjects with anosognosia had a lower GMD in the bilateral hippocampus ROI $(M=0.55, M d n=0.54$, $I Q R=0.06$, and $R n g=0.48-0.60)$ than subjects without anosognosia $(M=0.64, M d n=0.65, I Q R=0.08$, and $R n g=0.51-$ $0.73)$. This difference was statistically significant, showing a large effect size $\left(W=63.0, p=0.01\right.$, adjusted $p=0.04$, and $r_{r b}=0.75$, see Figure 2), and was accounted for by bilateral hippocampal differences (right hippocampus: anosognosia group $M=0.55$, $M d n=0.54, I Q R=0.07$, and $R n g=0.45-0.62$; non-anosognosia group $M=0.67, M d n=0.67 I Q R=0.12, R n g=0.55-0.77$, $W=64.0, p=0.01$, and $r_{r b}=0.78$; left hippocampus: anosognosia group $M=0.55, M d n=0.56, I Q R=0.05$, and $R n g=0.51-$ 0.57 ; non-anosognosia group $M=0.62, M d n=0.63, I Q R=0.06$, $R n g=0.48-0.69 W=62.0, p=0.01$, and $r_{r b}=0.72$ ).

The group of aMCI subjects with anosognosia also displayed a lower GMD in the bilateral cingulum cortex ROI $(M=0.47$, $M d n=0.47, I Q R=0.02$, and $R n g=0.44-0.51)$ than subjects without anosognosia $(M=0.51, M d n=0.51, I Q R=0.11$, and $R n g=0.43-0.60)$, but this difference was not statistically significant $\left(W=49.0, p=0.25\right.$, and $\left.r_{r b}=0.36\right)$. 
TABLE 2 | Demographic and clinical characteristics of patients with aMCl and AD displaying and not displaying anosognosia.

\begin{tabular}{|c|c|c|c|c|}
\hline & \multicolumn{2}{|c|}{$\mathrm{aMCl}(n=18)$} & \multirow[b]{2}{*}{$\begin{array}{c}\text { Test } \\
\text { statistic }\end{array}$} & \multirow[b]{2}{*}{$p$ value } \\
\hline & $\begin{array}{l}\text { Anosognosia } \\
(n=6)\end{array}$ & $\begin{array}{l}\text { No anosognosia } \\
(n=12)\end{array}$ & & \\
\hline $\begin{array}{l}\text { Age } \\
\text { (Years) }\end{array}$ & $\begin{array}{l}M=70.7 \\
M d n=72.0 \\
I Q R=3.0 \\
R n g=62.0-74.0\end{array}$ & $\begin{array}{l}M=68.8 \\
M d n=69.5 \\
I Q R=7.0 \\
R n g=60.0-75.0\end{array}$ & 27.5 & 0.45 \\
\hline $\begin{array}{l}\text { Sex } \\
\text { (Female, male) }\end{array}$ & 2,4 & 9,3 & 2.9 & 0.09 \\
\hline $\begin{array}{l}\text { Education } \\
\text { (Years) }\end{array}$ & $\begin{array}{l}M=12.8 \\
M d n=12.0 \\
I Q R=5.3 \\
R n g=6.0-17.0\end{array}$ & $\begin{array}{l}M=11.9 \\
M d n=12.0 \\
I Q R=7.0 \\
R n g=9.0-17.0\end{array}$ & 29.5 & 0.57 \\
\hline $\begin{array}{l}\text { MMSE } \\
\text { (Score) }\end{array}$ & $\begin{array}{l}M=26.3 \\
M d n=26.5 \\
I Q R=1.8 \\
R n g=25.0-28.0\end{array}$ & $\begin{array}{l}M=27.6 \\
M d n=28.0 \\
I Q R=1.3 \\
R n g=25.0-30.0\end{array}$ & 53.5 & 0.10 \\
\hline \multirow[t]{3}{*}{$\begin{array}{l}\text { ICV } \\
\left(\mathrm{cm}^{3}\right)\end{array}$} & $\begin{array}{l}M=1312.1 \\
M d n=1358.4 \\
I Q R=147.0 \\
R n g=1058.9- \\
1466.8\end{array}$ & $\begin{array}{l}M=1272.4 \\
M d n=1292.0 \\
I Q R=64.5 \\
R n g=1164.8 .0- \\
1397.2\end{array}$ & 25.0 & 0.34 \\
\hline & \multicolumn{2}{|c|}{$\mathrm{AD}(n=16)$} & & \\
\hline & $\begin{array}{l}\text { Anosognosia } \\
(n=7)\end{array}$ & $\begin{array}{l}\text { No anosognosia } \\
(n=9)\end{array}$ & $\begin{array}{c}\text { Test } \\
\text { statistic }\end{array}$ & $p$ value \\
\hline $\begin{array}{l}\text { Age } \\
\text { (Years) }\end{array}$ & $\begin{array}{l}M=71.6 \\
M d n=72.0 \\
I Q R=6.0 \\
R n g=65.0-76.0\end{array}$ & $\begin{array}{l}M=66.1 \\
M d n=64.5 \\
I Q R=6.0 \\
R n g=60.0-76.0\end{array}$ & 14.0 & 0.07 \\
\hline $\begin{array}{l}\text { Sex } \\
\text { (Female, male) }\end{array}$ & 5,2 & 5,4 & 0.4 & 0.52 \\
\hline $\begin{array}{l}\text { Education } \\
\text { (Years) }\end{array}$ & $\begin{array}{l}M=11.4 \\
M d n=9.0 \\
I Q R=3.5 \\
R n g=9.0-19.0\end{array}$ & $\begin{array}{l}M=12.6 \\
M d n=12.0 \\
I Q R=7.0 \\
R n g=6.0-20.0\end{array}$ & 38.5 & 0.47 \\
\hline $\begin{array}{l}\text { MMSE } \\
\text { (Score) }\end{array}$ & $\begin{array}{l}M=21.3 \\
M d n=21.0 \\
I Q R=4.0 \\
R n g=17.0-26.0\end{array}$ & $\begin{array}{l}M=23.3 \\
M d n=22.0 \\
I Q R=4.0 \\
R n g=18.0-30.0\end{array}$ & 43.5 & 0.26 \\
\hline $\begin{array}{l}\text { ICV } \\
\left(\mathrm{cm}^{3}\right)\end{array}$ & $\begin{array}{l}M=1379.1 \\
M d n=1358.1 \\
I Q R=137.6 \\
R n g=1184.1- \\
1414.8\end{array}$ & $\begin{array}{l}M=1284.5 \\
M d n=1293.4 \\
I Q R=117.3 \\
R n g=1262.6- \\
1519.2\end{array}$ & 13.0 & 0.06 \\
\hline
\end{tabular}

Values are presented as means (M), medians (Mdn), interquartile ranges (IQR) and ranges (Rng). Test statistics and $p$ values for age, education, MMSE and ICV correspond to the Mann-Whitney $U$ test. Test statistic and $p$ value for sex corresponds to the Chi-square test. aMCl, Amnestic mild cognitive impairment; $A D$, Alzheimer's disease dementia; MMSE, Mini-Mental State Examination; ICV, Intracranial volume.

In the $\mathrm{AD}$ group, subjects with anosognosia had a lower GMD in the bilateral hippocampus ROI $(M=5.1, M d n=0.55$, $I Q R=0.25$, and $R n g=0.33-0.71)$ than aMCI subjects without anosognosia $(M=0.56, M d n=0.57, I Q R=0.13$, and $R n g=0.46-0.77)$, but this difference was not statistically significant $\left(W=39.0, p=0.47\right.$, and $\left.r_{r b}=0.24\right)$. In an exploratory analysis, we identified that the Mdn hippocampal GMD of aMCI subjects with anosognosia $(M=0.55, M d n=0.54, I Q R=0.06$, and $R n g=0.48-0.60)$ was not statistically different than that the whole subset of $\mathrm{AD}$ subjects $(M=0.54, M d n=0.56 \mathrm{IQR}=0.14$, $R n g=0.33-0.77, W=48.0, p=1.00$, and $\left.r_{r b}<0.01\right)$, this is illustrated in Figure 2.

Alzheimer's disease subjects with anosognosia also displayed a lower GMD in the bilateral cingulum cortex ROI $(M=0.41$, $M d n=0.43, I Q R=0.09$, and $R n g=0.34-0.48)$ than subjects without anosognosia $(M=0.43, M d n=0.45 I Q R=0.10$, and $R n g=0.29-0.53)$, but this difference was not statistically significant $\left(W=39.0, p=0.47\right.$, and $\left.r_{r b}=0.24\right)$.

\section{White Matter Fractional Anisotropy Comparisons}

No statistically significant differences in hippocampal FA were found between aMCI subjects with anosognosia $(M=0.29$, $M d n=0.31 I Q R=0.10$, and $R n g=0.21-0.37)$ and without anosognosia $(M=0.29, M d n=0.27, I Q R=0.10, R n g=0.20-0.37$, $W=33.5, p=0.85$, and $\left.r_{r b}=-0.07\right)$. This was also the case in the cingulum FA, with no significant differences between aMCI with anosognosia $(M=0.44, M d n=0.47 I Q R=0.06$, and $R n g=0.36-$ 0.53 ) and without anosognosia $(M=0.44, M d n=0.42 I Q R=0.09$, $R n g=0.36-0.53, W=31.5, p=0.71$, and $\left.r_{r b}=-0.13\right)$.

Subjects with $\mathrm{AD}$ had similar results, with no significant differences in hippocampal FA between the anosognosia $(M=0.25, M d n=0.25, I Q R=0.11$, and $R n g=0.15-0.39)$ and no anosognosia groups $(M=0.25, M d n=0.24 I Q R=0.04$, $R n g=0.16-0.32 W=28.0, p=0.91$, and $r_{r b}=0.01$ ), and also no significant differences in cingulum FA between the groups (anosognosia group $M=0.38, M d n=0.36, I Q R=0.06$, and $R n g=0.30-0.52 ;$ non-anosognosia group $M=0.41, M d n=0.40$, $I Q R=0.05, R n g=0.34-0.49, W=39.0, p=0.23$, and $\left.r_{r b}=0.39\right)$.

\section{White Matter Mean Diffusivity Comparisons}

No statistically significant differences were found in hippocampal MD between aMCI subjects with anosognosia $(M=7.31 \mathrm{e}-4$, $M d n=5.07 \mathrm{e}-4, I Q R=4.71 \mathrm{e}-4$, and $R n g=4.47 \mathrm{e}-4-8.07 \mathrm{e}-4)$ and without anosognosia $(M=7.25 \mathrm{e}-4, M d n=0.27, I Q R=0.10$, $R n g=0.20-0.37, W=30.0, p=0.62$, and $\left.r_{r b}=-0.17\right)$. This was also the case in the cingulum MD, with no significant differences between aMCI with anosognosia $(M=5.98 \mathrm{e}-4, M d n=4.97 \mathrm{e}-4$ $I Q R=2.43 \mathrm{e}-4$, and $R n g=4.52 \mathrm{e}-4-8.39 \mathrm{e}-4)$ and without anosognosia $(M=6.28 \mathrm{e}-4, M d n=6.35 \mathrm{e}-4 I Q R=3.05 \mathrm{e}-4$, $R n g=4.47 \mathrm{e}-4-8.07 \mathrm{e}-4, W=32.5, p=0.78$, and $\left.r_{r b}=-0.10\right)$.

In the $\mathrm{AD}$ group, no significant differences were found in hippocampal MD between the anosognosia $(M=8.53 \mathrm{e}-4$, $M d n=6.32 \mathrm{e}-4, I Q R=7.00 \mathrm{e}-4$, and $R n g=4.36 \mathrm{e}-4-1.00 \mathrm{e}-3)$ and no anosognosia groups $(M=9.67 \mathrm{e}-4, M d n=9.56 \mathrm{e}-4$ $I Q R=2.39 \mathrm{e}-4, R n g=5.14 \mathrm{e}-4-2.00 \mathrm{e}-3 W=29.0, p=0.96$, and $r_{r b}=0.04$ ), and also no significant differences in cingulum $\mathrm{MD}$ between the groups (anosognosia group $M=6.81 \mathrm{e}-4$, $M d n=5.83 \mathrm{e}-4, I Q R=3.47 \mathrm{e}-4$, and $R n g=4.63 \mathrm{e}-4-$ $9.10 \mathrm{e}-4$; non-anosognosia group $M=7.34 \mathrm{e}-4, M d n=7.91 \mathrm{e}-4$, $I Q R=1.28 \mathrm{e}-4, R n g=4.78 \mathrm{e}-4-8.53 \mathrm{e}-4, W=26.0, p=0.86$, and $\left.r_{r b}=-0.07\right)$. 


\section{A Hippocampus GMD}

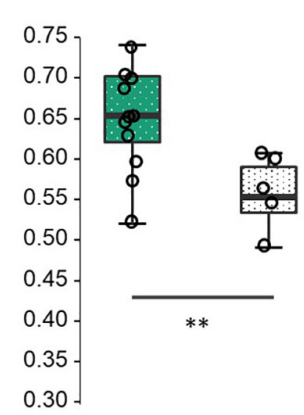

C Hippocampus FA

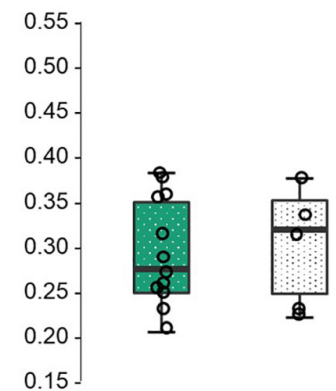

E Hippocampus MD

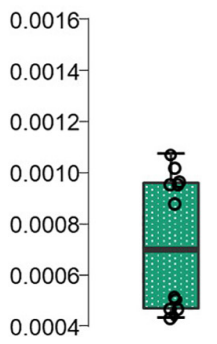

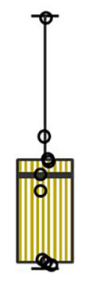
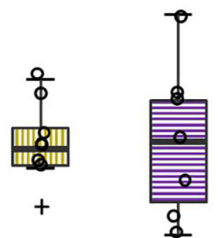

$+$
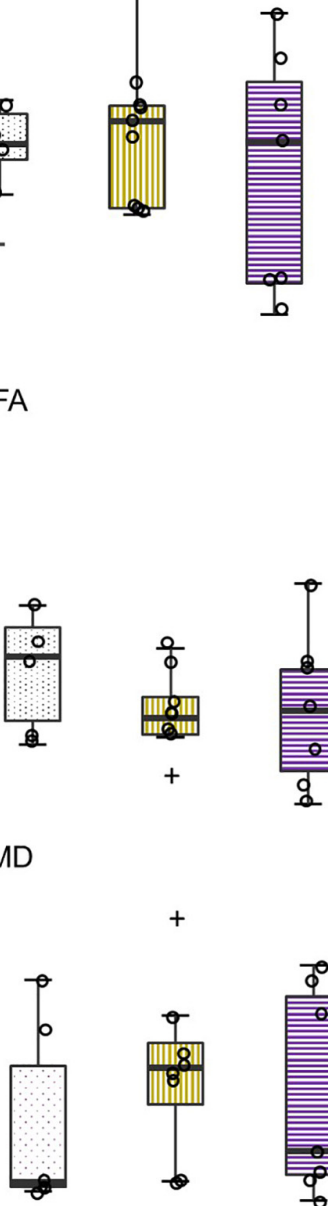

B Cingulum GMD

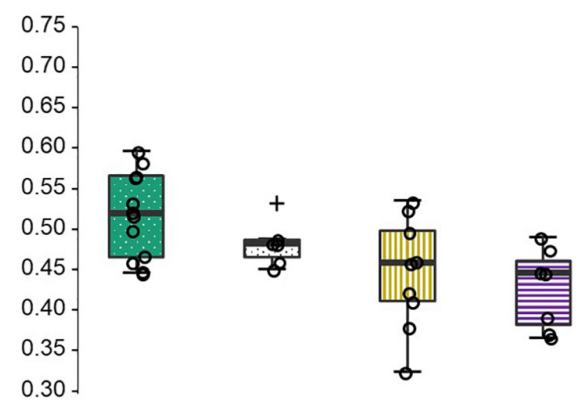

D Cingulum FA

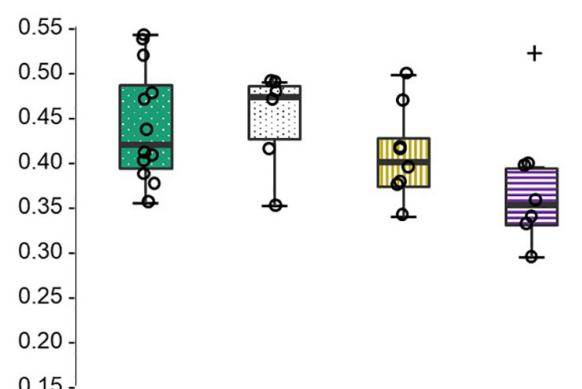

F Cingulum MD
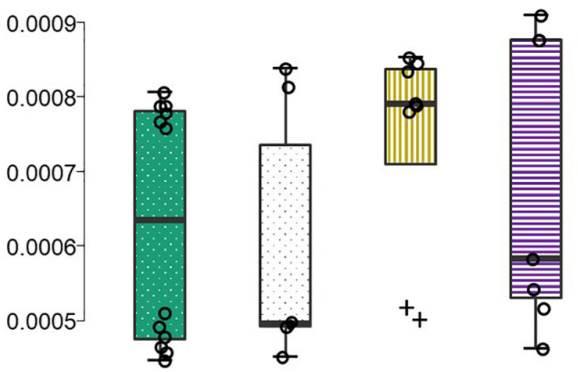

aMCl - anosognosia (-)
aMCl - anosognosia (+)
Alzheimer - anosognosia (-)
Alzheimer - anosognosia (+)

FIGURE 2 | Gray matter density in the hippocampal and cingulum regions of interest in aMCl subjects with and without anosognosia, and AD dementia participants. aMCl subjects with anosognosia displayed significantly less hippocampal gray matter density than aMCl subjects without anosognosia ( $\left.{ }^{\star \star} p=0.01\right)$. The hippocampal gray matter density of aMCl subjects with anosognosia was not statistically different from participants with AD dementia. Boxplots represent mean group values and interquartile ranges, outliers (values outside the lower of upper limits of the quartile range) are represented with the symbol +. GMD, gray matter density; FA, fractional anisotropy; MD, mean diffusivity; aMCl, amnestic mild cognitive impairment; AD, Alzheimer's disease. Although most of these correlations were not significant, there are trends shown in Supplementary Figure 1 that could inform future research, particularly the negative correlation between anosognosia scores and FA in both ROls shown in AD participants. If replicated, this suggests that white-matter integrity disturbances could result in a more pronounced anosognosia presentation in AD.

\section{DISCUSSION}

One of the main findings in this study, where we assessed volumetric and white matter tract changes associated with anosognosia, is that a group of aMCI subjects displaying anosognosia had a lower hippocampal volume than a group of aMCI subjects without anosognosia. These groups did not differ significantly by age, sex distribution, MMSE score, or ICV, which suggests that these groups were comparable. However, even more interestingly, aMCI subjects with anosognosia had a similar hippocampal volume to subjects with $\mathrm{AD}$ in our study. In the following, we will discuss the implications in the clinical 
characterization of aMCI and anosognosia assessment, neural and cognitive mechanisms underlying anosognosia, limitations of our study, and suggest future lines of research.

First, taking a clinical perspective, the association of reduced hippocampal volume with the presence of anosognosia in aMCI is in line with previous studies that place anosognosia as a risk factor for progression to AD dementia (Spalletta et al., 2014; Scherling et al., 2016). Hippocampal atrophy is independently and strongly correlated to AD progression from its early stages and to a heightened risk of progression from aMCI to AD dementia (Izzo et al., 2020; Zhuo et al., 2021). This implies that in clinical practice, an aMCI patient presenting to consultation with anosognosia might have a higher risk of progressing to dementia due to $\mathrm{AD}$, and could benefit from a closer follow-up, a more comprehensive diagnostic workup, and potentially, diseasemodifying strategies. Interestingly, our findings were obtained through a straight-forward classification of anosognosia. By using the screening questions of the BDSI (Godefroy et al., 2010), which are presented in Table 1, we dichotomized subjects as presenting and not presenting anosognosia, which is a simple approach that could be easily undertaken in everyday clinical assessments.

Regarding the neural and cognitive mechanisms underlying anosognosia, it has been hypothesized that because subjects affected by $\mathrm{AD}$ suffer from an inability to form new memories, they depend on remote personal semantics to evaluate their present performance, with inadequate self-appraisal (Morris and Mograbi, 2013; Vannini et al., 2017; Tondelli et al., 2018). This might explain why in our study anosognosia was significantly related to localized volumetric changes in the hippocampus, a fundamental structure for the formation of episodic memories (Eichenbaum, 2017). On the other hand, it is worth noting that $\mathrm{AD}$ subjects with anosognosia also displayed lower hippocampal and cingulum cortex volumes than $\mathrm{AD}$ subjects without anosognosia, but this difference was not statistically significant. This might imply that anosognosia in aMCI is an appropriate clinical marker of a brain phenotype that is within the $\mathrm{AD}$ continuum, but that anosognosia in $\mathrm{AD}$ arises from more subtle brain changes not limited to the hippocampus.

Some limitations of the current study are worth commenting on. The small sample size warrants caution in the interpretation of the results, which are thus in need of replication in larger samples. In addition, this implies that more subtle brain changes related to anosognosia might not have been detected. Another limitation that might explain the lack of differences in white matter tracts in our sample, is the concern that diffusion methods such as FA might be insufficient to study structures such as the cingulum bundle, where crossing-fiber anatomy might necessitate more specific measurements that track white matterfibers more reliably (Douaud et al., 2011; Jeurissen et al., 2014).

Our findings are in contrast to recent studies that identify a link between poor awareness of memory performance and a loss of white-matter integrity in the corpus callosum, frontalstriatal fibers and anterior thalamocortical radiations, as well as in the full right hemisphere (Bertrand et al., 2021; Chang et al., 2021). Beyond the fact that the number of ROIs used in the current study is limited by a small sample size, it is worth noting that the definition of anosognosia used in each study (different assessments of awareness of memory performance vs. structured questionnaire of everyday behavior) limits the comparability of the findings.

In sum, our preliminary findings, if replicated, suggest that anosognosia might be a relevant clinical marker for the suspicion of structural brain changes within the $\mathrm{AD}$ continuum in subjects with aMCI. Future studies assessing larger populations are necessary in order to contribute both to the characterization of aMCI subtypes and the understanding of the neural changes underlying anosognosia.

\section{DATA AVAILABILITY STATEMENT}

The raw data supporting the conclusions of this article will be made available by the authors, without undue reservation.

\section{ETHICS STATEMENT}

The studies involving human participants were reviewed and approved by National Institute of Neurology and Neurosurgery Mexico City. The patients/participants provided their written informed consent to participate in this study.

\section{AUTHOR CONTRIBUTIONS}

JF-V: study design, data collection, statistical design, data processing and analysis, and drafting of the manuscript. GR-G: data processing and analysis, and drafting of the manuscript. OM-M: study design, data collection, and the manuscript revision. RA-L: statistical design and the manuscript revision. ML: study design, statistical design, and the manuscript revision. YR-A: study design and data collection. GA-C: study design and data analysis. RR: study design and the manuscript revision. AA: study design, acquisition of funding, and the manuscript revision. SE-G: theoretical background, study design, and the manuscript revision. AS-O: theoretical background, study design, acquisition of funding, and drafting of the manuscript. All authors contributed to the article and approved the submitted version.

\section{FUNDING}

JF-V is a doctoral student from Programa de Doctorado en Ciencias Médicas, Odontológicas y de la Salud at the Universidad Nacional Autoìnoma de México (UNAM) in a joint program with the University of Groningen, Netherlands (RUG), and received a fellowship (number 465686, CVU 670327) from CONACYTMéxico and the RUG. This study received funding from the University Medical Centre, Groningen, Netherlands.

\section{SUPPLEMENTARY MATERIAL}

The Supplementary Material for this article can be found online at: https://www.frontiersin.org/articles/10.3389/fnagi.2021. 739422/full\#supplementary-material 


\section{REFERENCES}

Avants, B. B., Tustison, N. J., Wu, J., Cook, P. A., and Gee, J. C. (2011). An open source multivariate framework for n-tissue segmentation with evaluation on public data. Neuroinformatics 9, 381-400. doi: 10.1007/s12021-011-9 $109-y$

Bertrand, E., van Duinkerken, E., Laks, J., Dourado, M. C. N., Bernardes, G., Landeira-Fernandez, J., et al. (2021). Structural Gray and White Matter Correlates of Awareness in Alzheimer's Disease. J. Alzheimers Dis. 81, 13211330. doi: 10.3233/jad-201246

Chang, Y.-L., Chao, R.-Y., Hsu, Y.-C., Chen, T.-F., and Tseng, W.-Y. I. (2021). White matter network disruption and cognitive correlates underlying impaired memory awareness in mild cognitive impairment. NeuroImage Clin. 30:102626. doi: 10.1016/j.nicl.2021.102626

Chavoix, C., and Insausti, R. (2017). Self-awareness and the medial temporal lobe in neurodegenerative diseases. Neurosci. Biobehav. Rev. 78, 1-12. doi: 10.1016/ j.neubiorev.2017.04.015

Coupé, P., Yger, P., Prima, S., Hellier, P., Kervrann, C., and Barillot, C. (2008). An optimized blockwise nonlocal means denoising filter for 3-D magnetic resonance images. IEEE Trans. Med. Imaging 27, 425-441. doi: 10.1109/tmi. 2007.906087

Desikan, R. S., Ségonne, F., Fischl, B., Quinn, B. T., Dickerson, B. C., Blacker, D., et al. (2006). An automated labeling system for subdividing the human cerebral cortex on MRI scans into gyral based regions of interest. Neuroimage 31, 968-980. doi: 10.1016/j.neuroimage.2006.01.021

Douaud, G., Jbabdi, S., Behrens, T. E. J., Menke, R. A., Gass, A., Monsch, A. U., et al. (2011). DTI measures in crossing-fibre areas: increased diffusion anisotropy reveals early white matter alteration in MCI and mild Alzheimer's disease. Neuroimage 55, 880-890. doi: 10.1016/j.neuroimage.2010.1 2.008

Douaud, G., Smith, S., Jenkinson, M., Behrens, T., Johansen-Berg, H., Vickers, J., et al. (2007). Anatomically related grey and white matter abnormalities in adolescent-onset schizophrenia. Brain 130, 2375-2386. doi: 10.1093/brain/ awm 184

Eichenbaum, H. (2017). Prefrontal-hippocampal interactions in episodic memory. Nat. Rev. Neurosci. 18, 547-558. doi: 10.1038/nrn.2017.74

Flores-Vazquez, J. F., and Sosa-Ortiz, A. L. (2016). Mexican cross-cultural adaptation/validation of the "behavioral dysexecutive syndrome inventory.". Alzheimers Dement. J. Alzheimers Assoc. 12:818. doi: 10.1016/j.jalz.2016.06. 1660

Gerretsen, P., Chung, J. K., Shah, P., Plitman, E., Iwata, Y., Caravaggio, F., et al. (2017). Anosognosia is an independent predictor of conversion from mild cognitive impairment to Alzheimer's disease and is associated with reduced brain metabolism. J. Clin. Psychiatry 78, 1187-1196. doi: 10.4088/JCP. $16 \mathrm{~m} 11367$

Godefroy, O., Azouvi, P., Robert, P., Roussel, M., LeGall, D., and Meulemans, T. (2010). Dysexecutive syndrome: diagnostic criteria and validation study. Ann. Neurol. 68, 855-864. doi: 10.1002/ana.22117

Godefroy, O., Martinaud, O., Verny, M., Mosca, C., Lenoir, H., Bretalut, E., et al. (2014). The dysexecutive syndrome of alzheimer's disease: the grefex study. J. Alzheimers Dis. 42, 1203-1208. doi: 10.3233/jad-14 0585

Grieder, M., Wang, D. J. J., Dierks, T., Wahlund, L.-O., and Jann, K. (2018). Default mode network complexity and cognitive decline in mild Alzheimer's disease. Front. Neurosci. 12:770. doi: 10.3389/fnins.2018.00770

Hallam, B., Chan, J., Costafreda, S. G., Bhome, R., and Huntley, J. (2020). What are the neural correlates of meta-cognition and anosognosia in Alzheimer's Disease? A systematic review. Neurobiol. Aging 94, 250-264. doi: 10.1016/j. neurobiolaging.2020.06.011

Hanseeuw, B. J., Scott, M. R., Sikkes, S. A. M., Properzi, M., Gatchel, J. R., Salmon, E., et al. (2020). Evolution of anosognosia in alzheimer's disease and its relationship to amyloid. Ann. Neurol. 87, 267-280. doi: 10.1002/ana.2 5649

IBM Corp (2020). IBM SPSS Statistics for Windows, Version 27.0. Armonk, NY: IBM Corp.

Izzo, J., Andreassen, O. A., Westlye, L. T., and van der Meer, D. (2020). The association between hippocampal subfield volumes in mild cognitive impairment and conversion to Alzheimer's disease. Brain Res. 1728:146591. doi: 10.1016/j.brainres.2019.146591

JASP Team (2020). JASP (Version 0.14.1)[Computer software].

Jenkinson, M., Beckmann, C. F., Behrens, T. E. J., Woolrich, M. W., and Smith, S. M. (2012). Fsl. Neuroimage 62, 782-790. doi: 10.1016/j.neuroimage.2011.09. 015

Jeurissen, B., Tournier, J.-D., Dhollander, T., Connelly, A., and Sijbers, J. (2014). Multi-tissue constrained spherical deconvolution for improved analysis of multi-shell diffusion MRI data. Neuroimage 103, 411-426. doi: 10.1016/j. neuroimage.2014.07.061

Kerby, D. S. (2014). The simple difference formula: an approach to teaching nonparametric correlation. Compr. Psychol. 3, 11-15.

Mak, E., Chin, R., Ng, L. T., Yeo, D., and Hameed, S. (2015). Clinical associations of anosognosia in mild cognitive impairment and Alzheimer's disease. Int. J. Geriatr. Psychiatry 30, 1207-1214. doi: 10.1002/gps.4275

Manjón, J. V., Coupé, P., Martí-Bonmatí, L., Collins, D. L., and Robles, M. (2010). Adaptive non-local means denoising of MR images with spatially varying noise levels. J. Magn. Reson. Imaging 31, 192-203. doi: 10.1002/jmri.2 2003

McKhann, G. M., Knopman, D. S., Chertkow, H., Hyman, B. T., Jack, C. R., Kawas, C. H., et al. (2011). The diagnosis of dementia due to Alzheimer's disease: recommendations from the National Institute on Aging-Alzheimer's Association workgroups on diagnostic guidelines for Alzheimer's disease. Alzheimers Dement. 7, 263-269. doi: 10.1016/j.jalz.2011.0 3.005

Mondragón, J. D., Maurits, N. M., Deyn, P. P., De Deyn, P. P., and Alzheimer's Disease Neuroimaging Initiative (2021). Functional connectivity differences in Alzheimer's disease and amnestic mild cognitive impairment associated with AT(N) classification and anosognosia. Neurobiol. Aging 101, 22-39. doi: 10. 1016/j.neurobiolaging.2020.12.021

Morris, R. G., and Mograbi, D. C. (2013). Anosognosia, autobiographical memory and self knowledge in Alzheimer's disease. Cortex 49, 1553-1565. doi: 10.1016/ j.cortex.2012.09.006

Ostrosky-Solís, F., López-Arango, G., and Ardila, A. (2000). Sensitivity and specificity of the Mini-Mental State Examination in a Spanish-speaking population. Appl. Neuropsychol. 7, 25-31. doi: 10.1207/S15324826AN0 701_4

Petersen, R. C. (2004). Mild cognitive impairment as a diagnostic entity. J. Intern. Med. 256, 183-194. doi: 10.1111/j.1365-2796.2004.01388.x

Scherling, C. S., Wilkins, S. E., Zakrezewski, J., Kramer, J. H., Miller, B. L., Weiner, M. W., et al. (2016). Decreased self-appraisal accuracy on cognitive tests of executive functioning is a predictor of decline in mild cognitive impairment. Front. Aging Neurosci. 8:120. doi: 10.3389/fnagi.2016.0 0120

Smith, S. M., Jenkinson, M., Johansen-Berg, H., Rueckert, D., Nichols, T. E., Mackay, C. E., et al. (2006). Tract-based spatial statistics: voxelwise analysis of multi-subject diffusion data. Neuroimage 31, 1487-1505. doi: 10.1016/j. neuroimage.2006.02.024

Spalletta, G., Piras, F., Piras, F., Sancesario, G., Iorio, M., Fratangeli, C., et al. (2014). Neuroanatomical correlates of awareness of illness in patients with amnestic mild cognitive impairment who will or will not convert to Alzheimer's disease. Cortex 61, 183-195. doi: 10.1016/j.cortex.2014.1 0.010

Starkstein, S. E. (2014). Anosognosia in Alzheimer's disease: diagnosis, frequency, mechanism and clinical correlates. Cortex 61, 64-73. doi: 10.1016/j.cortex.2014. 07.019

Therriault, J., Ng, K. P., Pascoal, T. A., Mathotaarachchi, S., Kang, M. S., Struyfs, H., et al. (2018). Anosognosia predicts default mode network hypometabolism and clinical progression to dementia. Neurology 90, e932-e939. doi: 10.1212/ WNL.0000000000005120

Tondelli, M., Barbarulo, A. M., Vinceti, G., Vincenzi, C., Chiari, A., Nichelli, P. F., et al. (2018). Neural correlates of Anosognosia in Alzheimer's disease and mild cognitive impairment: a multi-method assessment. Front. Behav. Neurosci. 12:100. doi: 10.3389/fnbeh.2018.0 0100

Turró-Garriga, O., Garre-Olmo, J., Vilalta-Franch, J., Conde-Sala, J. L., de Gracia Blanco, M., and López-Pousa, S. (2013). Burden associated with the presence 
of anosognosia in Alzheimer's disease. Int. J. Geriatr. Psychiatry 28, 291-297. doi: 10.1002/gps.3824

Vannini, P., Hanseeuw, B., Munro, C. E., Amariglio, R. E., Marshall, G. A., Rentz, D. M., et al. (2017). Anosognosia for memory deficits in mild cognitive impairment: insight into the neural mechanism using functional and molecular imaging. NeuroImage Clin. 15, 408-414. doi: 10.1016/j.nicl.2017.0 5.020

Wakana, S., Caprihan, A., Panzenboeck, M. M., Fallon, J. H., Perry, M., Gollub, R. L., et al. (2007). Reproducibility of quantitative tractography methods applied to cerebral white matter. Neuroimage 36, 630-644. doi: 10.1016/j.neuroimage. 2007.02.049

Zamboni, G., Drazich, E., McCulloch, E., Filippini, N., Mackay, C. E., Jenkinson, M., et al. (2013). Neuroanatomy of impaired self-awareness in Alzheimer's disease and mild cognitive impairment. Cortex 49, 668-678. doi: 10.1016/j. cortex.2012.04.011

Zhuo, J., Zhang, Y., Liu, Y., Liu, B., Zhou, X., Bartlett, P. F., et al. (2021). New trajectory of clinical and biomarker changes in sporadic Alzheimer's disease. Cereb. Cortex 31, 3363-3373. doi: 10.1093/cercor/bhab017
Conflict of Interest: The authors declare that the research was conducted in the absence of any commercial or financial relationships that could be construed as a potential conflict of interest.

Publisher's Note: All claims expressed in this article are solely those of the authors and do not necessarily represent those of their affiliated organizations, or those of the publisher, the editors and the reviewers. Any product that may be evaluated in this article, or claim that may be made by its manufacturer, is not guaranteed or endorsed by the publisher.

Copyright (๑ 2021 Flores-Vázquez, Ramírez-García, Marrufo-Meléndez, AlcaláLozano, Lietz, Rodríguez-Agudelo, Acosta-Castillo, Renken, Aleman, EnriquezGeppert and Sosa-Ortiz. This is an open-access article distributed under the terms of the Creative Commons Attribution License (CC BY). The use, distribution or reproduction in other forums is permitted, provided the original author(s) and the copyright owner(s) are credited and that the original publication in this journal is cited, in accordance with accepted academic practice. No use, distribution or reproduction is permitted which does not comply with these terms. 